ISSN 1112-9867

http://www.jfas.info

\title{
STUDY OF OPPORTUNITIES OF USE OF COMPOSTS CUNICOLES FOR THE ABOVEGROUND PRODUCTION OF TOMATO PLANTS IN TUNISIA
}

\author{
Y. M'Sadak*, A. Bouallegue \\ Institut Supérieur Agronomique de Chott-Mariem, BP 47, CP 4042, Université de Sousse, \\ Tunisie
}

Received: 19 December 2014 / Accepted: 06 March 2015 / Published online: 07 March 2015

\begin{abstract}
The present work aims to study the potential valorization of composts exhausted cunicoles for aboveground vegetable plants. In a device complete random block with three repetitions, five composts in a pure state or in mixture and a witness are tested under tomato in seedbed except ground. They got results enable us to confirm that the composts are mature. As a whole, they have availability out of high water and a content of relatively weak air, whereas the substrates containing the peat-compost mixtures have physical properties close to the standards retained in Tunisia. The vegetative behavior of these plants with respect to the variation of the composition and the average size of the particles of the substrates shows a sensitivity of the seedlings to these parameters to the beginning of their growth. Majority of the mixtures containing peat-compost gave seedlings of quality, healthy, and homogeneous. The composts prepare well with a partial use in except ground.
\end{abstract}

Keywords: Composts; porosity; thermal behavior; vegetative behavior; tomato plants.

Author Correspondence, e-mail: msadak.youssef@yahoo.fr ICID: 1142897 


\section{INTRODUCTION}

L'appauvrissement des sols et la valorisation des déchets ainsi que leur élimination physique restent un enjeu vital pour la planète [1]. Judicieusement adapté aux conditions locales et géré de façon scientifique, le traitement de la fraction organique des déchets par le compostage contribuerait à résoudre ces difficultés [2]. Le compost est un produit stable et homogène, apte à enrichir les sols et à en restaurer la fertilité. En général, il est obtenu à partir de la dégradation biologique contrôlée de matière organique variée comme : feuilles mortes, déchets de bois, épluchures, déchets d'animaux morts, déjections [3]. Le compostage permet aux agriculteurs de faire face à la hausse des prix des engrais de même qu'il diminue les risques sanitaires posés par les décharges aux abords des grandes villes [4]. Les avantages agronomiques liés à l'utilisation des composts sont nombreux. Les principaux avantages apportés par l'utilisation des composts en plein champ sont directement liés à leur teneur en matière organique, à leur capacité à générer de l'humus stable [5], et à la stimulation de l'activité microbienne du sol et à celle des microorganismes du compost lui-même [6]. L'effet positif des composts est d'autant plus évident lorsque le sol est intensivement cultivé ou déséquilibré [7-9].

Le présent travail ne se propose pas d'évaluer la valorisation des composts en tant qu'amendement des sols. Il s'intéresse plutôt à l'étude des possibilités d'incorporation intégrale ou partielle des composts (après et avant extraction, autrement dit, épuisé ou non) dans la confection des substrats de culture (généralement à base de tourbe importée) pour la production hors sol des plants maraîchers, et plus précisément, des plants de tomate. La culture de tomate étant classée parmi les cultures stratégiques en Tunisie, vu la demande accrue tout au long de l'année, et surtout durant la saison estivale [10].

Diverses investigations ont dévoilé les potentialités d'utilisation des composts dans la production des plants forestiers [11-13]. Les déchets organiques, après compostage, peuvent être usagés comme substrat de croissance pour remplacer la tourbe [14-17]. C'est ainsi que de nombreux travaux récents ont mis l'accent sur le compostage des divers matériaux organiques disponibles localement [18] pour une valorisation organique dans les pépinières. Les matériaux couramment employés sont les résidus forestiers [19], les déchets agricoles [20], les déchets de jardin [21] et les déjections animales [22]. De même, plusieurs études ont démontré l'efficacité 
de l'emploi du compost en mélange avec la tourbe comme substrat de croissance pour la production de plants en pépinière [23-26]. Ainsi, l'objectif ultime de la présente étude est la substitution (partielle ou intégrale) de la tourbe importée par un mélange (Tourbe + Compost), destiné à l'élevage des plants de tomate, en ayant recours particulièrement aux résidus de l'extraction des composts, dénommés composts épuisés.

\section{MATÉRIEL ET MÉTHODES}

\subsection{Matériel}

L'étude a été conduite au printemps de l'année 2007 dans une serre expérimentale vitrée relevant du domaine de l'Institut Supérieur Agronomique de Chott-Mariem, Sousse, Tunisie. Cinq substrats, à base de compost pur, ont fait l'objet de l'étude (Essai 1) ; le compost cunicole brut $\left(\mathrm{CC}^{*} \mathrm{~B}\right)$, le compost cunicole brut épuisé ( $\left.\mathrm{CC} * \mathrm{BE}\right)$, le Co-Compost brut épuisé (CCBE), le Co-Compost affiné épuisé (CCAE) et le tamisat Co-Compost épuisé (TCCE) et cinq autres substrats à base de tourbe en mélange avec le compost (Essai 2) : (3/4T+1/4 CC*B (M1), 3/4T+1/4 CC*BE (M2), 3/4T+1/4 CCBE (M3), 3/4T+1/4 CCAE (M4) et 3/4T+1/4TCCE (M5)).

La préparation des composts a été réalisée par [27] à partir du compostage réalisé sur la plate-forme de la société «Lapins du Sahel » de Ksibet Sousse, Sousse, Tunisie. Les substrats ont été remplis dans des sacs en plastique souple de 10 à $15 \mathrm{~kg}$. Ils ont été stockés durant 10 mois à la température ambiante dans un bâtiment obscure et étanche aux pluies. Les quatre types de composts étudiés ont subi des extractions aérobiques durant 21 jours d'incubation. Un biotest de germination a été réalisé, selon la méthode de [28], pour contrôler la maturité des substrats et prévoir l'existence éventuelle de quelques éléments phytotoxiques [29] tout en mettant en considération la photosensibilité des semences de la plante- test [30]. Il s'agit de la laitue dans le cas considéré. Le suivi de la température journalière au niveau des conteneurs (placés sous abri) comportant différents substrats a été effectué à l'aide des thermomètres en parallèle avec le suivi de la température du sol sous le même abri chaque deux heures et durant cinq jours pour : $\left(\mathrm{CC} * \mathrm{BE}, \mathrm{CC}^{*} \mathrm{BE}, \mathrm{T}\right.$, et $\left.\mathrm{Sol}\right)$ et deux jours pour : (CCBE, TCCE, et CCAE). L'objectif ainsi recherché étant de caractériser leur comportement thermique. 


\subsection{Méthodes}

Le dispositif expérimental utilisé lors de cette étude est celui d'un plan Blocs aléatoires complets à un seul facteur avec répétitions (un facteur étudié avec six niveaux et un facteur contrôlé avec trois niveaux).

La semence utilisée est la tomate (Lycospersicum esculenta), variété (RIO GRAND). Les semences n'ont subi aucun traitement avant le semis, et le semis a été effectué directement dans des conteneurs multipots de 15 cavités (350 ml par cavité) placés sur des planches surélevées de $10 \mathrm{~cm}$ par rapport au sol. L'irrigation est effectuée tous les trois jours.

Deux paramètres physiques ont été fixés pour l'évaluation directe des substrats mis à l'essai :

(1) La porosité, elle exprime le volume d'air emprisonné dans le volume de compost [31] et est comprise entre $30 \%$ et $60 \%$ de façon à ce qu'on ait une bonne oxygénation, une bonne activité biologique ainsi qu'une bonne évacuation de l'eau, de la chaleur et du $\mathrm{CO}_{2}$ formé. La porosité totale $(\mathrm{Pt})$ est déterminée par le rapport entre le volume versé à la saturation de compost en eau et le volume total (équation I). La porosité d'aération $(\mathrm{Pa})$ est déterminée par le rapport entre le volume ressuyé à travers les trous de drainage sous l'effet des forces de gravité et le volume total (équation II).

La porosité de rétention (Pr) est déterminée par différence entre la porosité totale et la porosité d'aération (équation III). Dans les conditions tunisiennes, les valeurs retenues sont : Pt $\geq 50 \%$, $\mathrm{Pa} \geq 20 \%$ et $\operatorname{Pr} \geq 30 \%$, inspirées des normes canadiennes [32]. Les formules utilisées pour calculer les trois porosités et les méthodes adaptées dans le contexte Tunisien sont présentés ci-après. Il convient de noter que chaque test élémentaire de porosité était réalisé sur deux gobelets par répétition $=$ six gobelets par test élémentaire). Au total, 126 (six gobelets et 21 tests). Les 21 tests ont concerné six substrats purs (Tourbe et compost : Témoin et quatre composts épuisés) et 15 mélanges (trois ratios $\times$ cinq composts).

$P t=(V v / V t) \times 100$

$\mathrm{Pa}=(\mathrm{Vr} / \mathrm{Vt}) \times 100$

$\mathbf{P r}=\mathbf{P t}-\mathbf{P a}$

Avec : Vt : Volume total, Vv : Volume versé d'eau, Vr : Volume ressuyé.

(2) Le comportement thermique est effectué par suivi sous serre en plastique, régulièrement chaque deux heures des températures du sol à $20 \mathrm{~cm}$ de profondeur et des températures à l'intérieur des substrats empilés en conteneurs (350 ml par cavité), à travers ces mesures, nous 
avons suivi l'amplitude de variation des températures jour et nuit durant les mois de mars, avril et mai de l'année 2007.

L'évaluation indirecte a concerné la germination des semences et la croissance en hauteur des plants de tomate.

\subsection{Analyses statistiques}

Les données ont été analysées statistiquement par le logiciel SAS version 9.1.3, suivant les procédures décrites par [33]. L'analyse de la variance a été accomplie pour évaluer l'effet de chaque type de substrat sur le comportement végétatif (germination et croissance) des plants de tomate et la comparaison préliminaire de leur porosité ainsi que de leur comportement thermique.

\section{RÉSULTATS ET DISCUSSION}

\section{1. Évaluation directe des substrats}

\subsubsection{Maturité}

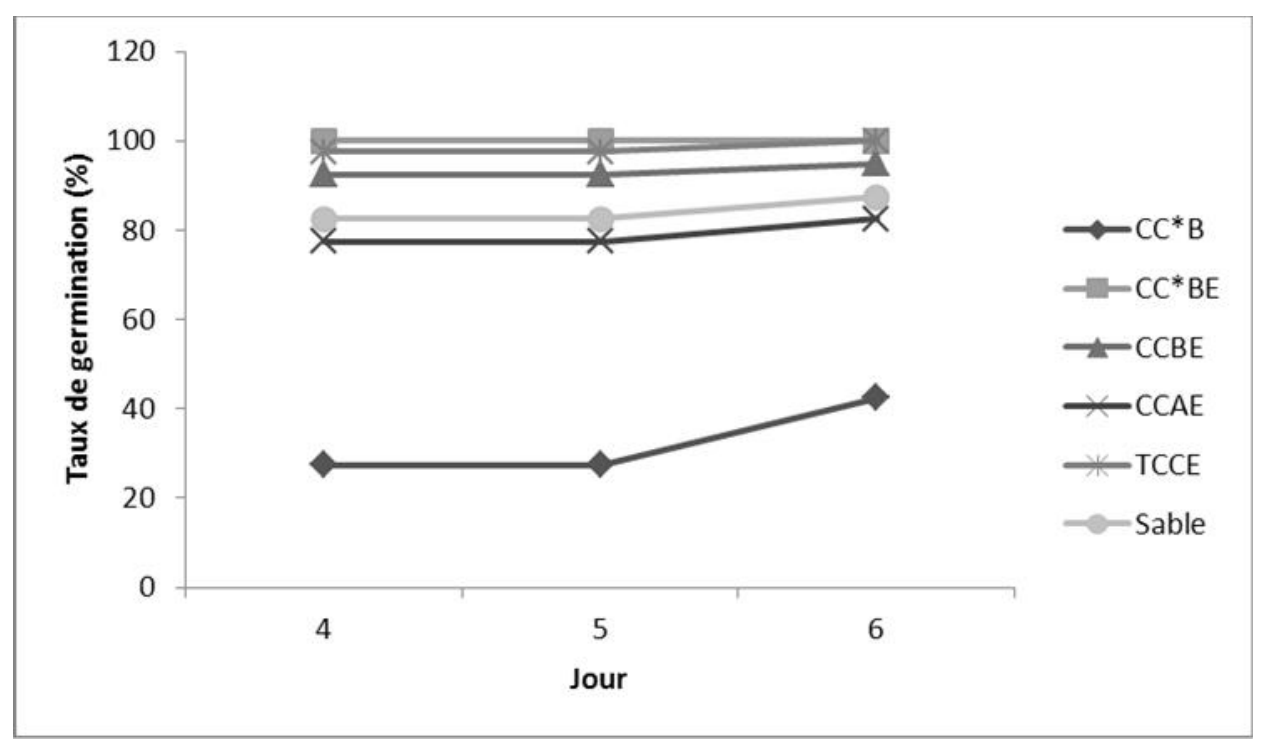

Fig.1. Évolution de la germination des semences de laitue lors du biotest

Les résultats des biotests de germination de la laitue sur les divers substrats de culture considérés et sur sable en tant que témoin (Figure 1) ont montré que les cinétiques relevées sont relativement identiques et les pourcentages de germination sont relativement différents chez les 
substrats utilisés. En effet, certains substrats ont même atteint un taux de $100 \%$ à partir du 4ème jour $(\mathrm{CC} * \mathrm{BE})$ et $(100 \%, 95 \%, 87,5 \%$ et $82,5 \%)$ le 6ème jour respectivement pour les substrats (TCCE, CCBE, Sable et CCAE), alors que le substrat CC*B a enregistré le taux de germination le plus faible (42,5\%), il s'agit, probablement, d'un substrat phytotoxique, ayant des substances inhibitrices de la germination. Selon [34], la plante réagit à ce milieu inhibiteur en diminuant sa respiration racinaire et son absorption d'une manière générale. De plus, elle ralentit sa production de gibbérelline, de cytokinine et l'ensemble de ses transports.

\subsubsection{Porosité}

Les résultats moyens de porosité tous substrats et mélanges confondus (Tableau 1) ne répondent pas aux normes de porosité d'aération ( $\mathrm{Pa} \geq 20 \%$ ), mais ils répondent aux normes de point de vue porosité de rétention et porosité totale, en se basant sur les normes de porosité adoptées en Tunisie (Pt $\geq 50 \%$ et $\mathrm{Pr} \geq 30 \%$ ).

Tableau 1. Porosités moyennes des substrats et mélanges étudiés

\begin{tabular}{ccccc}
\hline Variables & Moyenne & Ecart-type & Minimum & Maximum \\
\hline $\operatorname{Pt}(\%)$ & 77,4 & 7,01 & 66,6 & 89,1 \\
$\operatorname{Pa}(\%)$ & 18,7 & 3,35 & 15,3 & 25,1 \\
$\operatorname{Pr}(\%)$ & 58,7 & 3,88 & 51,3 & 64,0 \\
\hline
\end{tabular}

L'analyse de la variance montre que le type de substrat et la combinaison substrat-mélange ont un effet significatif sur les trois types de porosité (Pt, Pa et $\mathrm{Pr}$ ) au seuil de $\alpha=5 \% \quad(\mathrm{P}<0,001)$ avec un effet hautement significatif du mélange. En effet, les porosités totales les plus élevées sont enregistrées pour un même mélange chez le Co-Compost affiné épuisé (CCAE) avec une valeur moyenne de l'ordre de $84,5 \%$. Subséquemment le mélange (M1) a la porosité totale moyenne la plus élevée avoisinant $88 \%$.

Le test Duncan a permis de classer les substrats en quatre classes (Figure 2). En effet, le Co-Compost brut épuisé (CC*BE) et le tamisat Co-Compost épuisé (TCCE) se comportent de la même manière vis-à-vis des porosités totale et de rétention avec des valeurs moyennes 
respectivement $(72,2 \% ; 56,3 \%$ et $73,1 \% ; 56,1 \%)$, alors que le compost cunicole brut épuisé $(\mathrm{CC} * \mathrm{BE})$ et le Co-Compost brut épuisé (CCBE) ont presque la même porosité apparente, au seuil de $\alpha=5 \%$, avec des valeurs moyennes respectivement (56,3\% et $56,1 \%$ ).

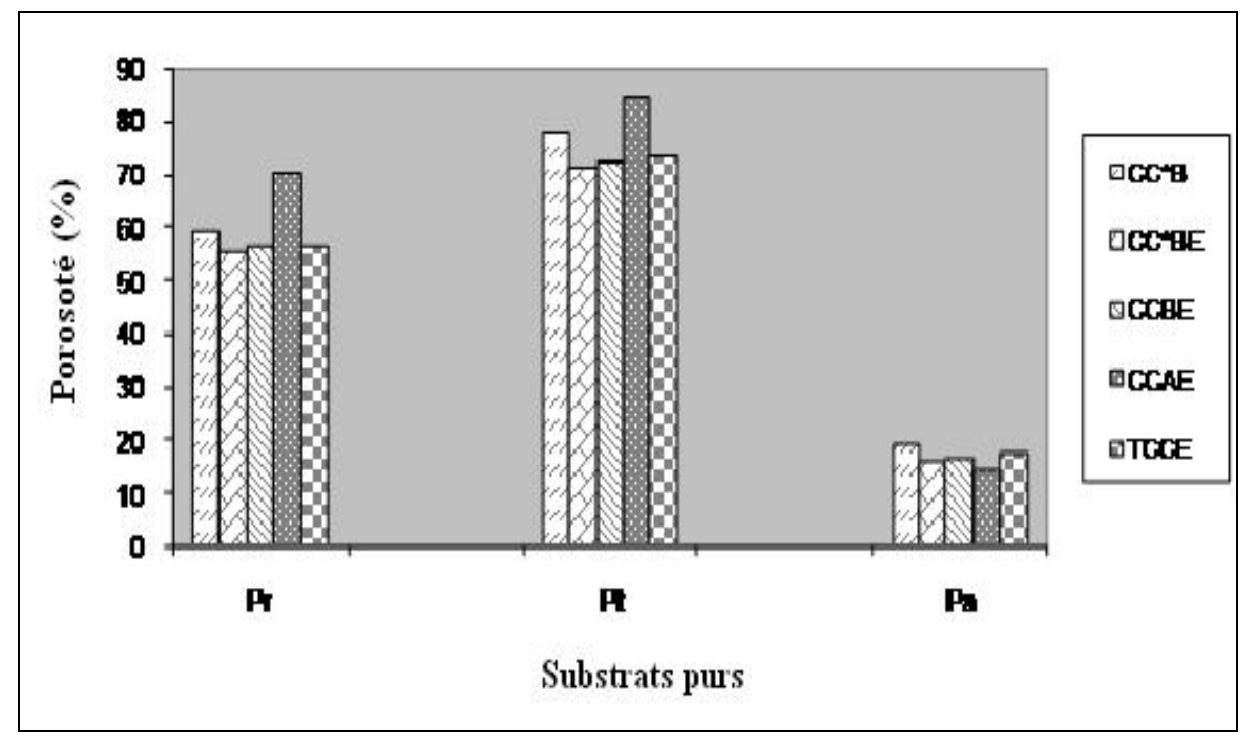

Fig.2. Porosités de différents substrats purs testés

Les mélanges, représentent chacun une classe à part (Figure 3). Cette différence provient des incidences directes du phénomène d'extraction sur les dimensions granulométriques des composts. En plus de la dégradation biologique lente par les microorganismes, l'eau peut agir directement par des réactions de dégradation et par des réactions d'hydrolyse. Ces dernières réduisent relativement la répartition granulométrique des composts. Notons ainsi que la capacité de rétention en eau peut augmenter et la porosité d'aération peut diminuer lorsque les composantes granulométriques des composts sont fines. Une bonne porosité s'avère nécessaire pour permettre à l'oxygène d'atteindre les racines et éviter ainsi la pourriture. Toutes les cellules vivantes, y compris celles des racines, ont besoin de l'oxygène pour la respiration et la croissance, et elles rejettent le dioxyde de carbone. Pour maintenir des niveaux adéquats d'oxygène et de dioxyde de carbone dans le substrat, l'échange de gaz avec l'atmosphère doit être garanti. Un contenu en oxygène supérieur à $12 \%$ dans le substrat ralentit l'apparition de nouvelles racines ; des niveaux de 5 et $10 \%$ sont très bas pour la croissance des racines établies ; et à des niveaux en dessous de 3\%, les racines ne se développent pas et finissent par flétrir. Des 
valeurs souhaitables de porosité totale qui maintiennent des niveaux d'oxygène au-dessus de $12 \%$ sont environ $50-80 \%$ par volume [35].

Partant de l'étude entreprise par [36], on peut dire que la confection des mélanges à base de tourbe et de Co-compost permet d'obtenir des substrats respectant dans une certaine mesure les normes standards de porosité et pouvant ainsi être un substitut partiel à la tourbe, engendrant une hémorragie moindre en matière de devises, tout en permettant de mieux valoriser les ressources locales en biomasse.

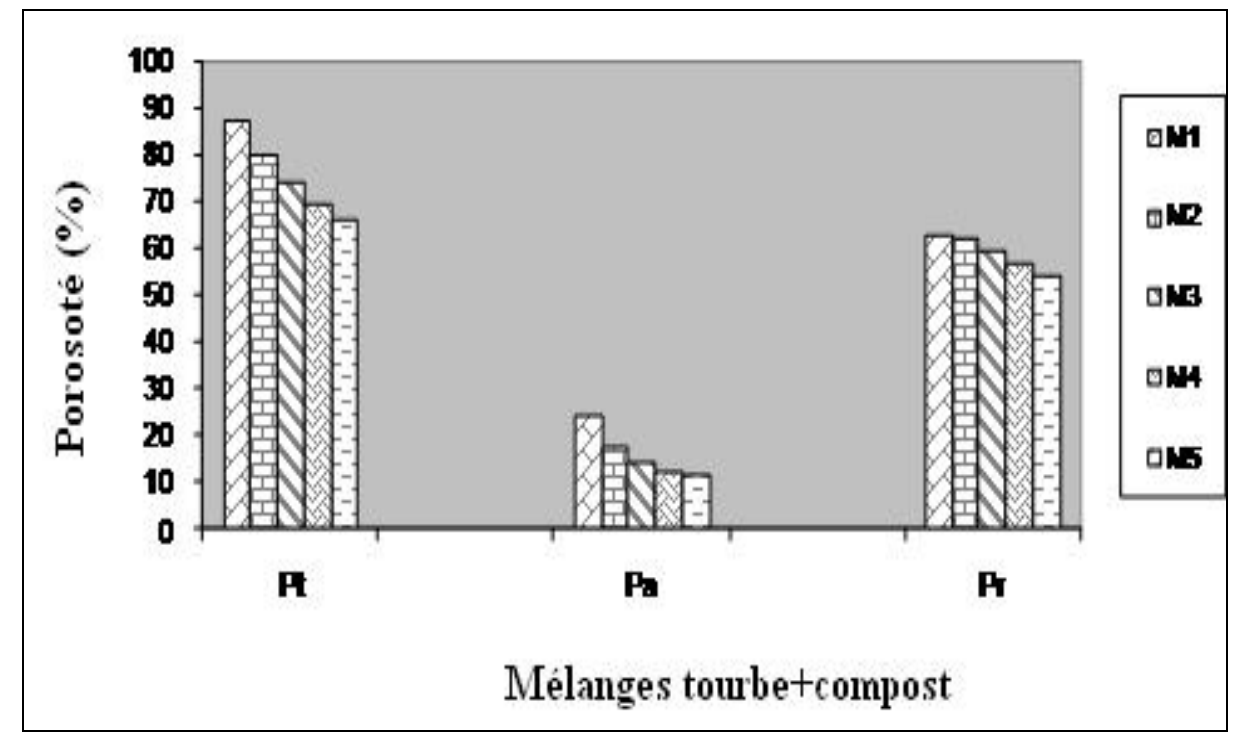

Fig.3. Porosités de différents mélanges mis à l'essai

\subsubsection{Comportement thermique}

Les températures, dans les divers composts étudiés ( $\mathrm{CC} * \mathrm{~B}, \mathrm{CC} * \mathrm{BE}$ et Tourbe), augmentent rapidement en comparaison avec celles dans le sol. Les températures les plus élevées sont enregistrées entre 12:00 H et 14:00 H où les rayonnements nets sont élevés avec des moyennes respectivement $\left(42,5,42,8\right.$, et $\left.46^{\circ} \mathrm{C}\right)$, alors que dans le sol, les températures maximales sont enregistrées à la fin de la journée entre 18:00 H et 20:00 H avec une température maximale $\left(24,5^{\circ} \mathrm{C}\right)$ (Figure 4$)$ 


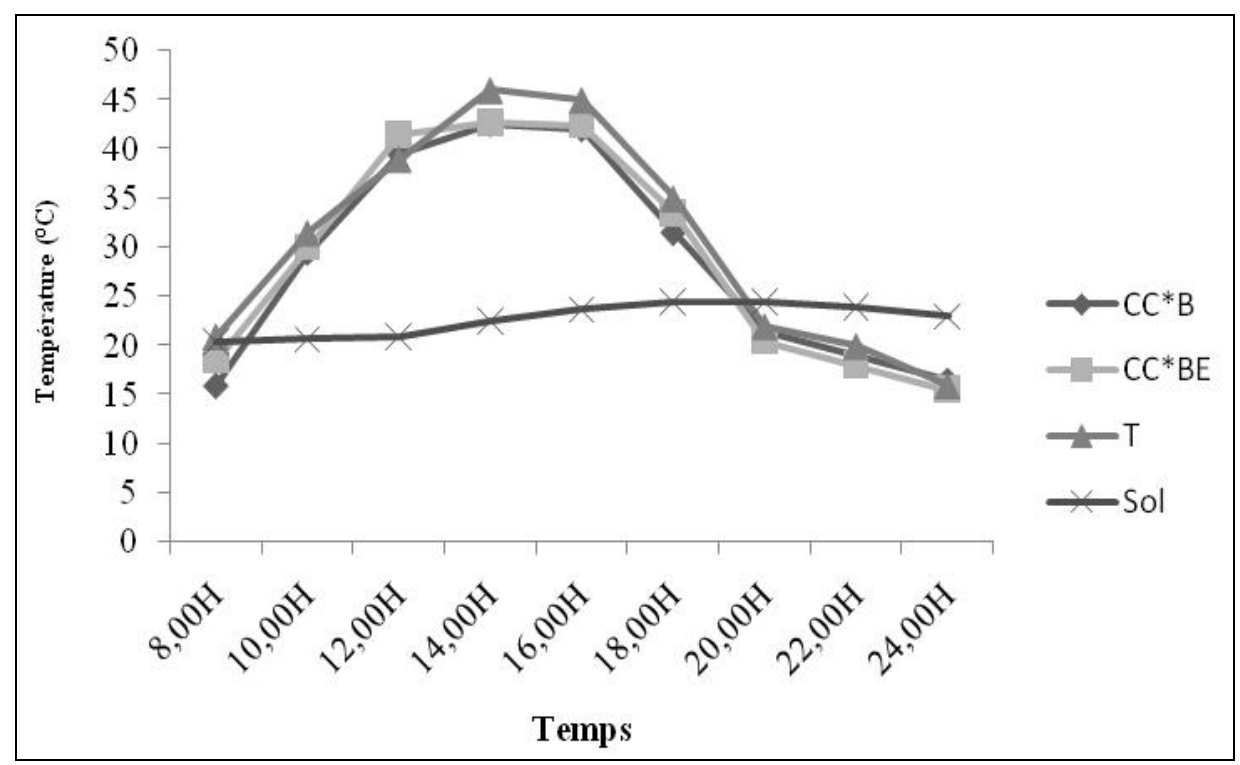

Fig.4. Évolution de la température des composts cunicoles durant une journée

Les résultats de températures relevées, pendant une journée, chez les Co-Composts considérés vérifient bien les résultats précédents. Il reste à signaler que la couleur des substrats pourrait renseigner sur les caractéristiques thermiques des composts. Plus les substrats sont opaques, plus ils s'échauffent plus vite. A cet égard, les températures les plus élevées sont mesurées dans la tourbe (T), suivie par le Co-Compost brut épuisé (CCBE) avec des températures respectivement de 43 et $39,5^{\circ} \mathrm{C}$ (Figure 5).

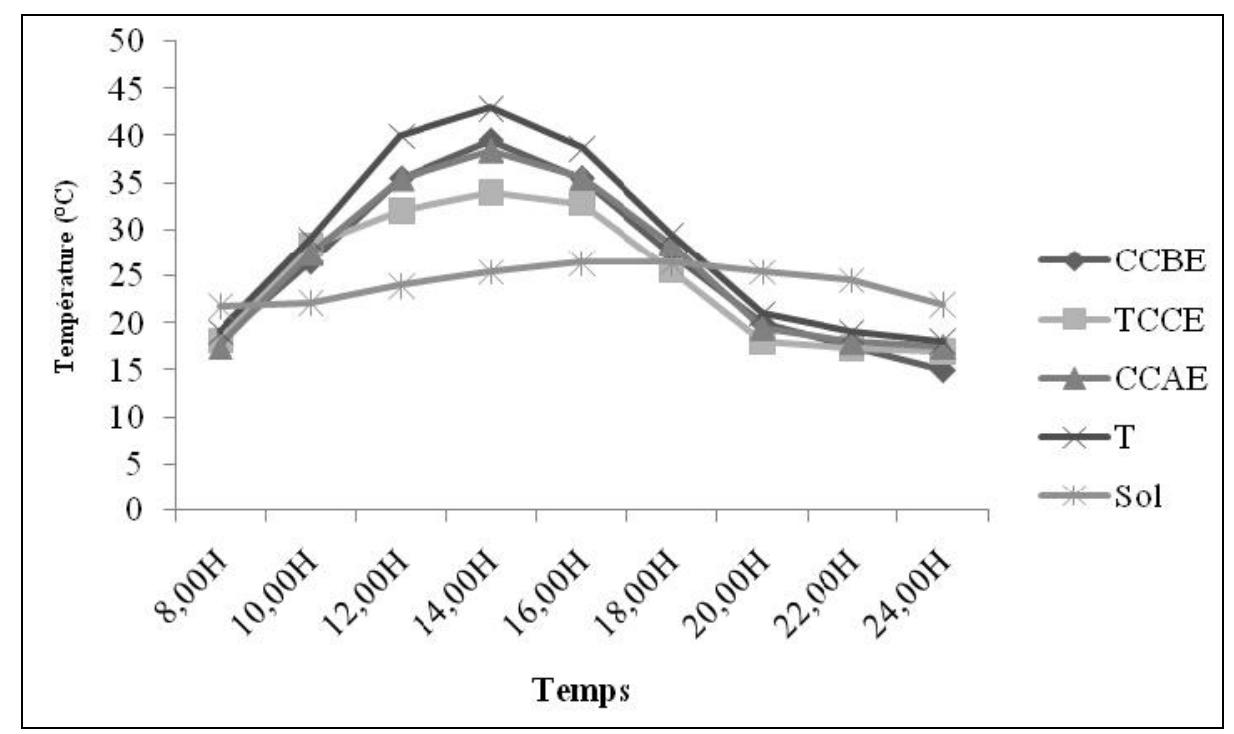

Fig.5. Évolution de la température des Co-Composts cunicoles durant une journée 


\section{2. Évaluation indirecte des substrats}

\subsubsection{Germination}

* Essai 1 : Résultats relatifs à la substitution totale

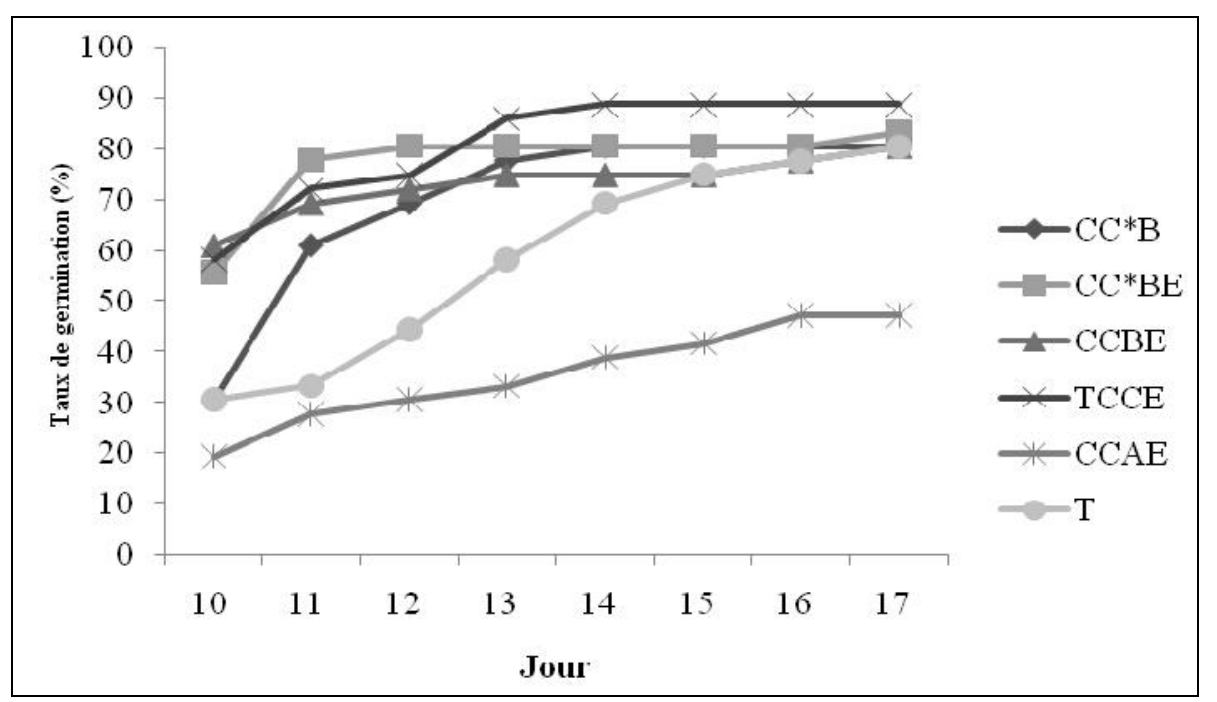

Fig.6. Effet des substrats à base de compost sur la germination des semences de tomate

Les résultats des essais de germination des semences de tomate sur les différents substrats à base de compost pur ont montré que les cinétiques et les pourcentages de germination dans les différents substrats testés ont été relativement identiques (Figure 6). Ces pourcentages ont varié de $83,3 \%$ chez le substrat CC*BE à $90 \%$ chez le substrat TCCE à l'exception du substrat CCAE qui a montré un taux de germination de l'ordre de 47,2\%, probablement à cause de sa faible porosité d'aération $(\mathrm{Pa}=9,3 \%)$ qui a un impact direct sur la germination. L'excellente germination indique que le compost ne contient pas de substances phénoliques qui peuvent entraver la germination des plants [37]. Ceci laisse suggérer que les différents types de compost sont prêts à être utilisés pour produire des plants à l'échelle opérationnelle dans les pépinières maraîchères.

* Essai 2 : Résultats relatifs à la substitution partielle

Concernant l'effet des mélanges (3/4 tourbe $+1 / 4$ compost) sur le processus de germination chez la tomate, les résultats trouvés ont montré des faibles taux de germination au début (après 10 jours de semis) et des taux germination élevés plus tard (après 18 jours). Il semble que les composts incorporés avec la tourbe ont un effet négatif : Retard de germination (Figure 7). 


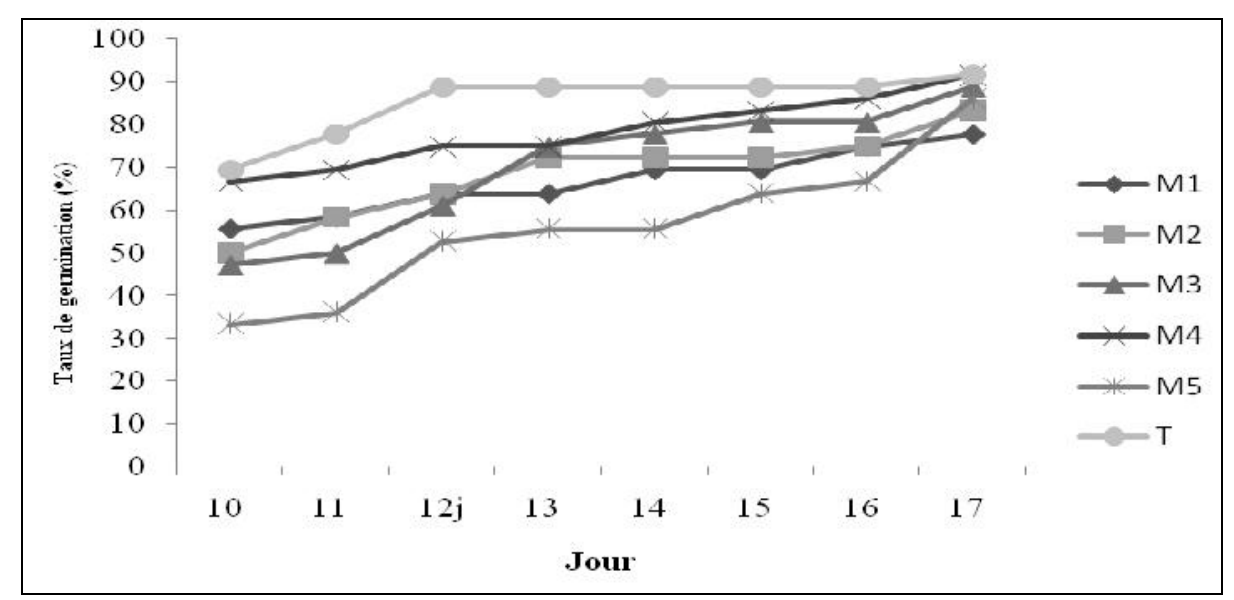

Fig.7. Effet des mélanges tourbe-compost sur la germination des semences de tomate

\subsubsection{Croissance des plants en hauteur}

* Essai 1 : Résultats relatifs à la substitution totale

A partir du diagnostic des courbes de la cinétique et la vitesse de croissance en hauteur des plants de tomate (Figure 8), on peut noter que les composts $\mathrm{CC}^{*} \mathrm{BE}$ et $\mathrm{CC} * \mathrm{~B}$ ont permis de produire les meilleurs plants de point de vue accroissement en hauteur en comparaison avec ceux installés sur les autres composts.

A ce propos, nous avons observé une stimulation de la croissance en hauteur et une augmentation plus ou moins importante du nombre de feuilles. Le développement des plants le plus faible est relevé pour le compost CCAE. Il semble que ce compost a une répercussion négative sur la croissance des organes végétatifs. Les hauteurs considérées des plants sont moins élevées que celles du témoin. L'émission de nouvelles feuilles a été également réduite ainsi que leurs tailles. Il est à noter que ces résultats sont souvent en relation avec les caractéristiques chimiques (une teneur élevée en éléments nutritifs peut exprimer une phytotoxicité des jeunes plants) et leurs caractéristiques physiques (une faible teneur en air peut provoquer une asphyxie qui implique la diminution de la croissance des jeunes plants comme, elle peut causer la disparition de ces derniers). Signalons enfin que la tourbe (témoin) constitue un substrat de croissance, par excellence, pour la production des plants de tomate et que sa substitution intégrale par un compost local ( épuisé ou non) n'est pas conseillée d'après les conditions expérimentales considérées. 


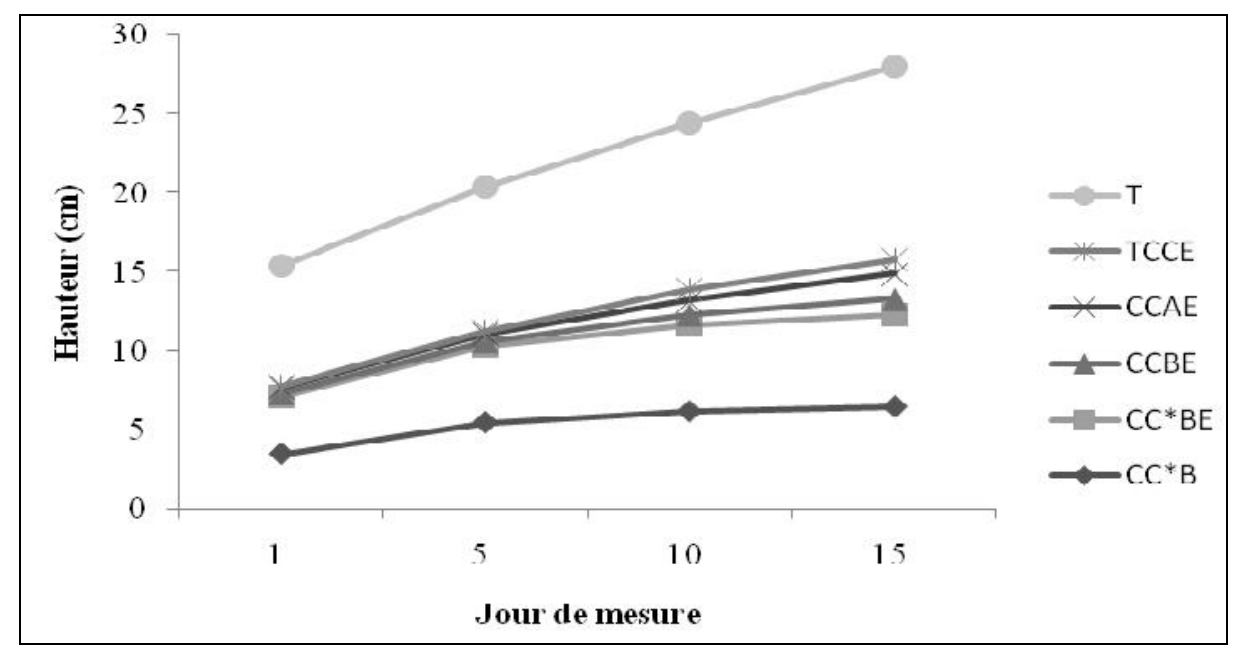

Fig.8. Effet des substrats à base de compost sur la croissance en hauteur des plants de tomate

\section{* Essai 2 : Résultats relatifs à la substitution partielle}

Les résultats relevés dévoilent que le compost incorporé avec la tourbe peut agir sur la croissance végétative. Les faibles apports $(25 \%)$ de compost dans le substrat à base de tourbe ont fourni des plants sains et de qualité.

Il convient de constater que la matière organique, en l'occurrence, le compost, agit sur les plants par ses effets directs (disponibilité des éléments nutritifs) et par ses effets indirects en tamponnant le substrat tourbeux (protection des plants contre les agents pathogènes). A cet égard, le ratio de mélange (3/4 tourbe $+1 / 4$ compost) pourrait être conseillé en pépinières maraîchères (Figure 9).

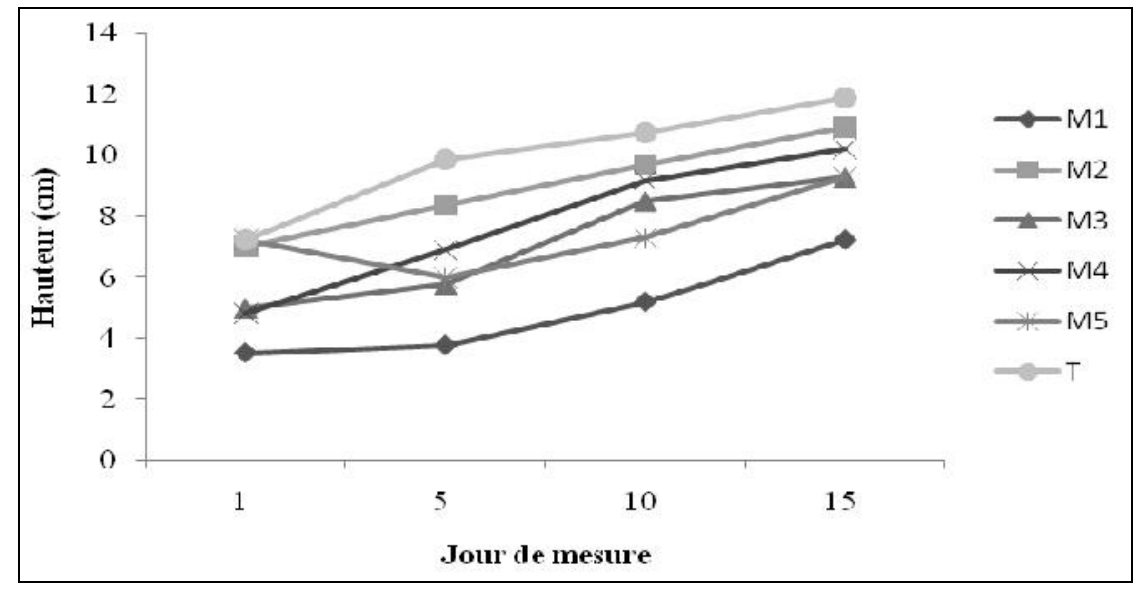

Fig.9. Effet des mélanges tourbe-compost sur la croissance en hauteur des plants de tomate 


\section{CONCLUSION}

Les résultats acquis lors de cette étude ont clairement démontré les potentialités d'utilisation des différents composts testés dans la composition des substrats pour l'élevage des plants maraîchers en pépinière hors sol. Cependant, il reste à caractériser davantage les propriétés physico-chimiques des substrats, ainsi que leurs effets sur la qualité des plants, notamment sur la physiologie et la croissance des parties aériennes et racinaires des plants produits.

Ainsi, il convient de préciser les deux enseignements importants tirés de deux essais mis en place pour la production hors sol des plants de tomate, à savoir :

- La substitution complète de la tourbe par un compost local (épuisé ou non) ne mérite pas d'être encouragée surtout dans la confection des substrats destinés pour la production des plants maraîchers.

- La substitution partielle de la tourbe par le compost (avant ou après extraction) à raison de $25 \%$, voire 30 à $40 \%$ pourrait constituer une alternative attirante pour les pépiniéristes, tout en permettant la réduction des importations de tourbe, voire d'autres intrants (produits phytosanitaires pour phytoprotection et/ou fertilisants pour fertigation des plants).

En définitive, des investigations futures doivent être poursuivies pour la mise au point de quelques substrats de culture à partir de matériaux organiques disponibles pour la production des plants en pépinière maraîchère.

\section{REMERCIEMENTS}

Les auteurs sont reconnaissants au Propriétaire de la Société Lapins du Sahel à Ksibet Sousse, gouvernorat de Sousse (Tunisie), pour avoir mis à leur disposition les composts et les Co-composts (à l'état épuisé ou non) mis à l'essai, comme substituts de la tourbe importée.

\section{RÉFÉRENCES BIBLIOGRAPHIQUES}

[1] Juste C. Avantages et inconvénients des composts d'ordures ménagères comme amendement organique des sols et supports de cultures. INRA, France, 1979, 27 p.

[2] Mustin M. Le compost- Gestion de la matière organique. Ed. François Dubusc, Paris, France, 1987, 954 p. 
[3] Sidoum H. Le compostage. Série Technologie Enda Tiers monde-Preceup, 1996, 17 p. www.globenet.org/preceup/fr/docsfr/sidoum.rtf

[4] Juste C., Solda P. Étude des possibilités d'utilisation des composts d'ordures ménagères comme supports de cultures maraîchères. INRA Bordeaux, MECV (Convention n 75-135), 1979, $22 \mathrm{p}+$ Annexes.

[5] Gobat J.M., Aragno M., Matthey W. Le Sol vivant : Bases de pédologie -Biologie des sols. Deuxième édition, Presses Polytechniques et Universitaires Romandes, 2003, 568 p.

[6] Craft C.M., Nelson E.B., Applied and Environmental Microbiology, 1996, 62, $1550-1557$.

[7] Fuchs J. G. Influence de la qualité biologique des composts sur les plantes et leur santé (AnnexeA1-A13). Biophyt AG. Institut de Recherches et de Consultations en Agronomie et Ecologie Appliquées, CH - 5465 Mellikon, 1996, 13 p.

www.biophyt.ch/documents/cours_paris.pdf

[8] Chausson P. Utilisation des composts en agriculture: Synthèse des essais-vitrines 1989-1998. Compost-Diffusion, Sol-Conseil, Suisse, 1999, 27 p.

[9] Fuchs J.G. Practical use of quality compost for plant health and vitality improvement. In: S. Klammer (ed.), Microbiology of Composting, Berlin Heiselberg: Springer-Verlag, 2002, 435-444.

[10]M'Sadak Y., Jelali R., Ali A., J. Fund. App. Sci., 2013, 5 (2), 167-176. jfas.info/index.php/JFAS/article/download/158/pdf

[11]Miller J.H., Jones N. Organic and compost-based growing media for tree seedlings nurseries. Washington, DC, États-Unis, Banque Mondiale, World Bank Technical Papers, $1995,264,75 \mathrm{p}$.

[12]Raviv M., Zaidman B. Z., Kapulnik Y., Compost Science \& Utilization, 1998, 6 (1), 46-52.

[13]Fitzpatrick G. E. Compost utilization in ornamental and nursery crop production systems. In: Compost utilization in horticultural cropping systems. Stoffella P. J., Kahn B. A. (éds). New York, États-Unis, Lewis Publishers, 2001, 135-150.

[14] Simins H.I., Manios V.L. Mixing peat with MSW compost. Biocycle 31, 1990, 60-61. 
[15]Pryce S. Alternative to peat. Professional Horticulture, 1991, 5, 101-106.

[16] García-Gómez A., Bernal M.P., Roig A., Bioresour. Technol., 2002, 83, 81-87.

[17] Benito M., Masaguer A., De Antonio R., Moliner A., Bioresour. Technol., 2005, 96, 597-603.

[18] Abad M, Noguera P, Burés S., Bioresour. Technol., 2001, 77, 197-200.

[19] Veijalainen A.M., Juntunen M.L., Heiskanen J., Lilja A., Scand. J. Forest. Res., 2007, $22,390-397$.

[20] Manios T., Environ. Int., 2004, 29, 1079-1089.

[21] Brewer L.J., Sullivan D.M., 2003, 11 (2), 96-112.

[22] Desalegn G, Binner E, Lechner P., Compos. Sci. Util., 2008, 16 (2), 90-98.

[23] Sanderson K.C., Hortic. Sci., 1980, 15, 173-178.

[24] Roe N.E., Kostewicz S.R. Germination and early growth of vegetable seed in compost. Univ. Florida Proc., 1992, 101, 191-201.

[25] Purman J.R., Gouin F.R., J. Environ. Hortic., 1992, 10, 52-54.

[26] Inbar Y., Chen Y, Hoitink H.A.J. Properties for establishing standards for utilization of compost in container media, In: Hoitink HAJ, Keener HM (eds.). Science and engineering of composting: Design, environmental, microbiological and utilization aspects. Renaissance Publication, Worthington, OH, 1993, 668-694.

[27] Jelali R. Maîtrise de la fabrication et de la qualité des composts cunicoles. Projet de fin d'études, I.S.A. Chott Mariem, Sousse, Tunisie, 2006, 75 p.

[28] Spohn E. Determination of compost maturity. Compost Science/Land Utilization, 1978, 19(3), 26-27.

[29] Cole M. Simple chemical test for compost maturity. Recherche effectuée à l'Université de l'Illinois, cité dans Biocycle, 1992, p. 26.

[30] Spohn E., Compost Science, 1969, 24-26.

[31] Lardinois I., Van De Kludert A. Organic Waste. Ed.: Tool, Amsterdam, Hollande, 1993, $57-79$.

[32] CPVQ Pépinières, Culture en conteneurs, Substrats. Document technique, Conseil des Productions Végétales du Québec, Canada, 1993, 19 p. 
[33] Gomez K.A., Gomez R.A. Statistical Procedures for Agricultural Research. Second Edition, 1984, 657 p.

[34] Devisscher S. Propriétés et valorisation du compost. Mém. D.E.S.S., Univ. Picardie, France, 1997, 60 p. www.u-picardie.fr/beauchamp/duee/compost1.htm

[35] Hannah J. Bonnes pratiques de culture en pépinières forestières : Directives pratiques pour les pépinières de recherche. Manuel technique $\mathrm{n}^{\circ} 3$. World Agroforestry Centre (ICRAF), 2006, 93 p. www.worldagroforestry.org/downloads/publications/PDFs/mn14474.pdf

[36] M’Sadak Y., Elouaer M.A., Dhahri M., Revue Nature \& Technologie, 2013, N 9 (B), 27-34. www.univ-chlef.dz/revuenatec/Issue_09_Art_B_05.pdf

[37] Sullivan D.M., Miller R. O. Compost quality attributes, measurements, and variability. In: Stofella P.J. and Kahn, B.A. (Eds.). Compost utilization in horticultural cropping systems. Lewis Publishers, New York, USA, 2001, 95-120.

\section{How to cite this article:}

M'Sadak Y, Bouallegue A. Study of opportunities of use of composts cunicoles for the aboveground production of tomato plants in Tunisia. J. Fundam. Appl. Sci., 2015, 7(2), 244-259. 